\title{
Irreversible inhibition of BoNT/A protease: Unique warhead reactivity and function contingent upon a bifunctional approach
}

Lewis D. Turnere, ${ }^{\mathrm{a},}$, Alexander L. Nielsen ${ }^{\mathrm{a}, \mathrm{b},{ }^{*}, \text { Lucy Lin }^{\mathrm{a}} \text {, Sabine Pellett }}$, Takashi Sugane ${ }^{\mathrm{a}}$, Margaret E. Olson ${ }^{\mathrm{a}, \mathrm{d}}$ Eric A. Johnson ${ }^{\mathrm{c}}$, and Kim D. Janda ${ }^{\mathrm{a}, \mathrm{f}}$

\begin{abstract}
Botulinum neurotoxin A (BoNT/A) is categorized as a Tier 1 bioterrorism agent and persists within muscle neurons for months, causing paralysis. A readily available treatment that abrogates BoNT/A's toxicity and longevity is a necessity in the event of a widespread BoNT/A attack and for clinical treatment of botulism, yet remains an unmet need. Herein, we describe a comprehensive warhead screening campaign of bifunctional hydroxamate-based inhibitors for the irreversible inhibition of the BoNT/A light chain (LC). Using the 2,4dichlorocinnamic hydroxamic acid (DCHA) metal-binding pharmacophore modified with a pendent warhead, a total of 37 compounds, possessing 13 distinct warhead types, were synthesized and evaluated for timedependent inhibition against the BoNT/A LC. Iodoacetamides, maleimides, and an epoxide were found to exhibit time-dependent inhibition and their $k_{\mathrm{GSH}}$ measured as a description of reactivity. The epoxide exhibited superior time-dependent inhibition over the iodoacetamides, despite reacting with glutathione (GSH) 51-fold slower. The proximity-driven covalent bond achieved with the epoxide inhibitor was contingent upon the vital hydroxamate- $\mathrm{Zn}^{2+}$ anchor in placing the warhead in an optimal position for reaction with Cys165. Monofunctional control compounds exemplified the necessity of the bifunctional approach, and Cys165 modification was confirmed through high-resolution mass spectrometry (HRMS) and ablation of timedependent inhibitory activity against a C165A variant. Compounds were also evaluated against BoNT/Aintoxicated motor neuron cells, and their cell toxicity, serum stability, and selectivity against matrix metalloproteinases (MMPs) were characterized. The bifunctional approach allows the use of less intrinsically reactive electrophiles to intercept Cys165, thus expanding the toolbox of potential warheads for selective irreversible BoNT/A LC inhibition. We envision that this dual-targeted strategy is amenable to other metalloproteases that also possess non-catalytic cysteines proximal to the active-site metal center.
\end{abstract}

\footnotetext{
aDepartments of Chemistry and Immunology, The Skaggs Institute for Chemical Biology, Worm Institute of Research and Medicine (WIRM), Scripps Research, 10550 N Torrey Pines Road, La Jolla, CA 92037, United States.

${ }^{b}$ Center for Biopharmaceuticals \& Department of Drug Design and Pharmacology, Faculty of Health and Medical Sciences, University of Copenhagen, Universitetsparken 2, DK-2100, Copenhagen, Denmark.

${ }^{\mathrm{c}}$ Department of Bacteriology, University of Wisconsin, 1550 Linden Drive, Madison, WI 53706, United States.

${ }^{\mathrm{d} C}$ College of Pharmacy, Roosevelt University, Schaumburg, IL 60173, United States.

*These authors contributed equally to this work.

${ }^{\ddagger}$ Correspondence to: kdjanda@scripps.edu
} 


\section{Introduction}

Botulinum neurotoxins (BoNTs) are produced by a pathogenic Gram-positive, anaerobic bacterium known as Clostridium botulinum, and are amongst the most neurotoxic agents known. ${ }^{1}$ To date, seven serotypes of BoNT (A-G) have been identified, with serotypes A, B, E and F causing botulism in humans. ${ }^{2,3}$ BoNT/A has the highest incidence rate amongst its serotype counterparts and is the most toxic and causes the most severe botulism, ${ }^{4}$ with an estimated intravenous $\mathrm{LD}_{50}$ of $1-2 \mathrm{ng} / \mathrm{kg}$ and $\sim 10 \mathrm{ng} / \mathrm{kg}$ by inhalation. ${ }^{5}$ Furthermore, it has a half-life of several months once internalized in neurons. ${ }^{6}$ The toxicity, persistence, ease of production, and dissemination of BoNT/A, coupled with no effective post-intoxication remedy, has warranted its designation by the Centers for Disease Control and Prevention (CDC) as a Tier 1 bioterrorism agent alongside smallpox, plague and anthrax. ${ }^{7}$

In contrast to its potential misuse in bioterrorism, BoNT/A is used for a wide array of therapeutic applications. Disorders that are characterized by aberrant muscular spasticity have been a focus of BoNT/A treatment, including migraines, post-stroke injury, spinal cord injury and more recently, cerebral palsy. ${ }^{8-11}$ BoNT/A is also used cosmetically for a variety of aesthetic imperfections, with a staggering 7.7 million procedures carried out in 2019, up 878\% from the year 2000. ${ }^{12}$ The huge increase in BoNT/A cosmetic usage over the past two decades comes with health concerns; consistent and long-term use of BoNT/A has been associated with adverse side-effects including necrotizing fasciitis, respiratory compromise, iatrogenic botulism, and even anaphylactic shock, leading to death. ${ }^{13}$

Both the toxicity and the therapeutic potential of BoNT/A arise from its ability to disrupt vesicle fusion at neuronal termini, thus preventing acetylcholine release and synaptic transmission, which results in flaccid paralysis and botulism. ${ }^{14}$ The BoNT/A holotoxin is a large protein complex of $150 \mathrm{kDa}$ that consists of a heavy chain (HC, $100 \mathrm{kDa}$ ) and a $\mathrm{Zn}^{2+}$ metalloprotease light chain (LC, $50 \mathrm{kDa}$ ). The $\mathrm{HC}$ associates with the neuronal membrane through recognition of specific gangliosides and receptor proteins resulting in endocytosis of the toxin. ${ }^{15}$ Endosomal acidification results in conformation changes leading to membrane insertion of $\mathrm{N}$-terminus of the HC, which subsequently leads to translocation of the LC to the cytosol. ${ }^{16}$ Once internalized, the LC is released from the HC via disulfide bond reduction, and is able to selectively bind synaptosomal-associated protein 25 (SNAP-25) and implement site-specific cleavage through $\mathrm{Zn}^{2+}$-mediated proteolysis. This disrupts the action of the soluble N-ethylmaleimide sensitive factor (NSF) attachment protein receptor (SNARE) complex, a crucial component of vesicular fusion to the synaptosomal membrane. ${ }^{17}$

The potential misuse of BoNT/A in a wide-scale bioterrorism event, coupled with adverse events stemming from its prolific therapeutic and cosmetic use, means that a readily available, non-toxic, and effective reversal agent is vital. Currently, the only approved treatment for BoNT/A-induced botulism is an equinederived antitoxin, which targets and neutralizes the BoNT/A holotoxin before neuronal internalization has taken place. However, this treatment method has a limited therapeutic window (12-24 h post-intoxication) as the antibody-based antitoxin cannot enter neuronal compartments. ${ }^{18}$ This pitfall outlines the necessity to develop a treatment that can not only inhibit internalized BoNT/A but also address its longevity.

To this end, our group has discovered several examples of reversible small-molecule inhibitors of the BoNT/A LC, with the majority possessing metal-binding groups (MBGs) that disrupt SNAP-25 cleavage 
through coordination to the active site $\mathrm{Zn}^{2+} \cdot{ }^{19,20}$ One of the most potent inhibitors to date is DCHA (Fig.1 A) which exhibits a $K_{i}$ of $0.3 \mu \mathrm{M}$ against the BoNT/A LC. ${ }^{21}$ Despite displaying promising in vitro activity, and modest activity in vivo against BoNT/A-challenged mice, DCHA (and other reversible inhibitors) have not progressed beyond pre-clinical studies, ${ }^{22}$ due in part to the transient nature of reversible inhibition. Because of the longevity of the BoNT/A LC in neurons, a long-term consistent dosing regimen of a reversible inhibitor would need to be employed. Additionally, metalloenzyme inhibitors often exhibit cross-target inhibition and associated side-effects, meaning that dosing of such molecules over several months is not a viable strategy. ${ }^{23}$, 24

Irreversible inhibition has the potential to abrogate BoNT/A's toxicity and longevity by means of permanent deactivation. Cys165, which lies approximately 5-8 $\AA$ away from the $\mathrm{Zn}^{2+}$ center, has become the focus of several targeted covalent inhibition strategies. ${ }^{25-28}$ Stura et al. reported the first X-ray crystal structure of the BoNT/A LC covalently modified on Cys165 with (2-aminoethyl) methanethiosulfonate (MTSEA), a small molecule containing the highly reactive methanethiosulfonate (MTS) warhead. ${ }^{26}$ Recently, we sought to integrate both reversible and irreversible aspects in a bifunctional fashion, whereby DCHA was modified with a pendent MTS moiety that had the potential to simultaneously chelate the $\mathrm{Zn}^{2+}$ metal and react with Cys $165 .{ }^{27}$ An overlay (Fig. 1B) of both X-ray co-crystal structures of DCHA and MTSEA bound to the BoNT/A LC shows how the active site may simultaneously accommodate both a small covalent molecule and a reversible MBG inhibitor. Compound 1 (Fig. 1A) showed excellent enzyme inhibition with a $k_{\text {inact }} / K_{\mathrm{i}}$ value of $418 \mathrm{M}^{-1} \mathrm{~s}^{-}$ ${ }^{1}$. However, this promising biochemical data was not reflected in a BoNT/A-implicated cell assay. ${ }^{27}$ The exaggerated disconnect between the enzymatic and cellular efficacy likely stemmed from the promiscuous nature of the MTS warhead. ${ }^{27,29}$ To address this issue, we describe an extensive screening campaign in search of intrinsically less reactive warheads that were capable of forming proximity-driven covalent bonds with Cys165 for the irreversible inhibition of the BoNT/A LC.

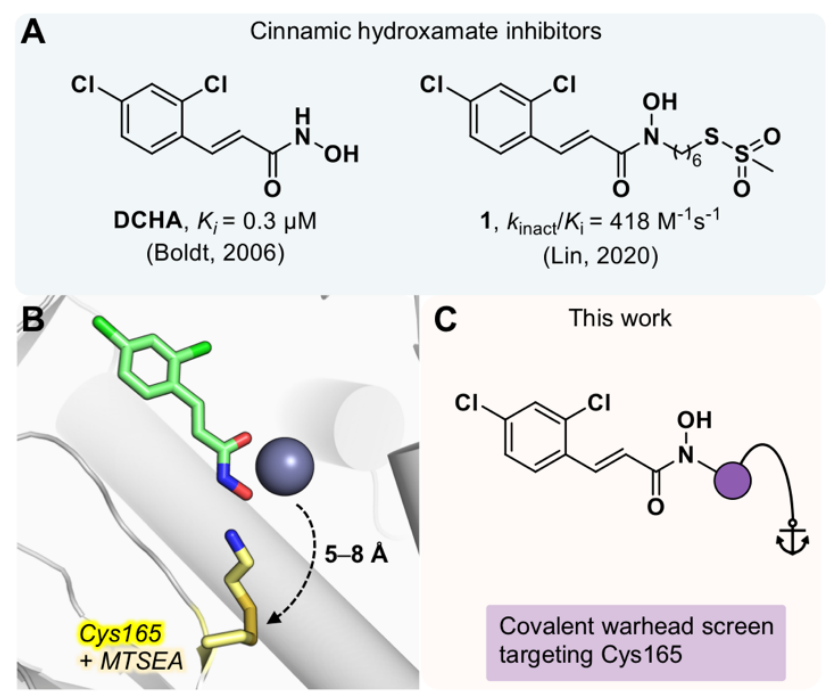

Fig. 1. (A) Previously reported BoNT/A inhibitors based on the cinnamic hydroxamate scaffold. (B) X-ray co-crystal structures outlining the basis for a bifunctional approach, overlaying DCHA (green; PDB 2IMA) ${ }^{28,30}$ and MTSEA (yellow; PDB 4ELC) ${ }^{26}$ bound to Cys 165 near the active site $\mathrm{Zn}^{2+}$ (purple sphere). (C) Overview of research explored in this project. 


\section{Results and Discussion}

\section{Compound design and synthesis}

Acrylamides are well known for their safety and selectivity profiles when forming selective, proximity-driven covalent bonds and therefore were selected for synthesis. An additional ten distinct warhead categories, possessing a variety of reactivities and sizes, were also synthesized (Fig. 2A). Beginning with 2,4dichlorocinnamic acid, $O$-tetrahydropyran (THP)-protected linkers possessing warhead precursor functionality, e.g. alkene, amine, alcohol, were coupled to the carboxylic acid using simple amide coupling conditions. Key chemical transformations such as amide couplings or Mitsunobu reactions ${ }^{31}$ were then performed to install the electrophilic species, with subsequent THP deprotection to afford the final hydroxamate compounds (Fig. 2A). In the case of condition-sensitive warheads such as the epoxides and aldehydes, deprotection occurred before functionalization of the reactive warhead (Scheme S5).

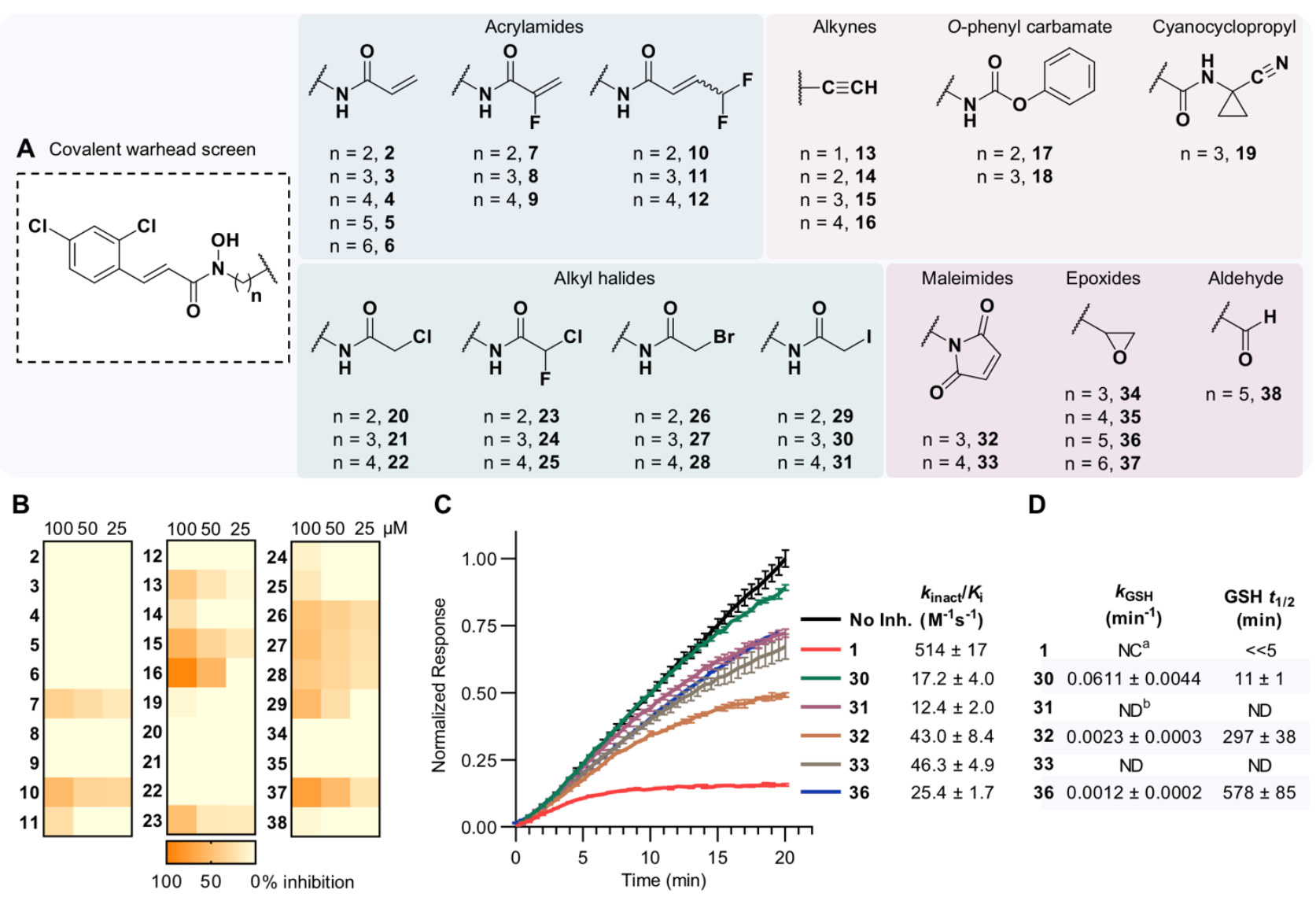

Fig. 2. (A) Summary of compounds synthesized. See ESI-1, Section 4.0 for full synthetic details. (B) Heatmap of BoNT/A LC inhibition exhibited by reversible compounds expressed as percent inhibition. Data for $\mathbf{1 7}$ and $\mathbf{1 8}$ omitted due to solubility issues. See Table S1 for full details. (C) Representative data from the continuous SNAPtide assay showing time-dependent inhibition of the BoNT/A LC from covalent compounds with their calculated $k_{\text {inact }} / K_{\mathrm{i}}$ values outlined. Displayed data was obtained at $37^{\circ} \mathrm{C}$ at a compound concentration of $32 \mu \mathrm{M}$, excluding 1 , which was screened at $8 \mu \mathrm{M}$. Data was normalized to the maximal fluorescence of the no inhibitor control at 20 min. Error bars represent \pm SD, $n=3$. See Fig. S1 for additional graphs. (D) GSH reactivity data for covalent hits. Errors reported as SEM, $\mathrm{n}=4$. See Fig. $\mathbf{S 2}$ for additional data. ${ }^{\mathrm{a}} \mathrm{NC}=$ not calculable, ${ }^{\mathrm{b}} \mathrm{ND}=$ not determined. 


\section{Optimization of warheads for irreversible inhibition}

Compounds were evaluated for time-dependent inhibition using a continuous SNAPtide fluorescence resonance energy transfer (FRET) assay, which allowed for facile identification of covalent inhibitors by their characteristic non-linear inhibition profile of BoNT/A LC activity. ${ }^{27}$ Acrylamides 2-12, alkynes 13-16, $O$ phenyl carbamates 17-18 and cyanocyclopropyl 19 did not exhibit time-dependent inhibition and, with the exception of the alkynes, appeared to inhibit the BoNT/A LC poorly (Fig. 2B). It was hypothesized that these warheads lacked the necessary reactivity to form a covalent bond with Cys165. Previous reports have postulated that Cys 165 possesses a low acidity and exists as a thiolate anion under physiological coniditions. ${ }^{25}$, ${ }^{28}$ To investigate this, we performed covalent docking of compound 2 with the BoNT/A LC (Fig. S3A) to predict if warheads larger than the MTS-warhead could be accommodated within the active site; the results clearly indicated that a covalent bond could be formed with Cys165. However, computational evaluation of protonation states using PROPKA ${ }^{32}$ predicted low nucleophilicity/basicity of Cys165 with a pKa of 12.6 (Fig. S3B) and could explain the lack of time-dependent inhibition observed for compounds 2-19.

Our predicted low reactivity of Cys165 suggested that warheads possessing higher reactivities were required to form the desired covalent bond. Thus, a series of haloacetamide (20-31) and maleimide (32-33) containing inhibitors were evaluated, as both groups are regularly used as cysteine capping reagents in bioconjugation. ${ }^{33,34}$ In fact, we observed that the less reactive chloro- and bromo-acetamides $\mathbf{2 0}-\mathbf{2 8}$ did not show time-dependent inhibition within the continuous assay, whereas iodo acetamides $\mathbf{3 0}$ and $\mathbf{3 1}$ exhibited moderate $k_{\text {inact }} / K_{\mathrm{i}}$ values of 17.2 and $12.4 \mathrm{M}^{-1} \mathrm{~s}^{-1}$, respectively (Fig. 2C). Although compound 29 also contained the iodoacetamide group, its shorter linker length may not have allowed simultaneous $\mathrm{Zn}^{2+}$ coordination and Cys165 engagement, thus preventing the covalent reaction. We have observed in the past that covalent bond formation with bifunctional inhibitors not only requires sufficient reactivity but also proximity of the warhead to Cys165. ${ }^{27}$ Maleimides 32 and $\mathbf{3 3}$ also exhibited time-dependent inhibition with slightly improved $k_{\text {inact }} / K_{\mathrm{i}}$ values of 43.0 and $46.3 \mathrm{M}^{-1} \mathrm{~s}^{-1}$, respectively. Intriguingly, the less reactive epoxide $\mathbf{3 6}$ displayed covalent inhibition with a $k_{\text {inact }} / K_{\mathrm{i}}$ value of $25.4 \mathrm{M}^{-1} \mathrm{~s}^{-1}$. The lack of time-dependent inhibition observed for compounds $\mathbf{3 5}$ and $\mathbf{3 7}$ emphasized the importance of linker length in the epoxide series, with $\mathbf{3 6}$ possessing optimum positioning for a proximity-driven covalent bond to form with Cys165. The subtle structure-activity relationship (SAR) for the epoxide series was not reflected in the iodoacetamides (30 and 31) and maleimides (32 and 33), with both compounds for each warhead type exhibiting equipotent time-dependent inhibition. This suggests that the requirements for successful covalent bond formation with Cys 165 for 30-33 was biased towards the inherent reactivity of the warheads as opposed to optimal alignment of the reactive vectors.

To directly measure reactivity differences between each warhead type, we quantified the GSH reactivity of compounds 1, 30, 32 and 36 (Fig. 2D). ${ }^{35}$ Notably, compound 1 reacted near-instantaneously with GSH, meaning the rate of depletion could not be confidently quantified. Compound $\mathbf{3 0}$ was also shown to be highly reactive with a $t_{1 / 2}=11 \mathrm{~min}$, while both 32 and $\mathbf{3 6}$ were less reactive towards GSH with $\mathrm{t}_{1 / 2} \sim 5 \mathrm{~h}$ and $10 \mathrm{~h}$, respectively. Moreover, epoxide-containing compound 36 reacted with GSH $\sim 51$-fold slower than iodoacetamide 30 but exhibited a superior $k_{\text {inact }} / K_{\mathrm{i}}$ value. This emphasizes the importance of Cys 165 proximity on the success of these bifunctional inhibitors; clearly the advantage conferred by having higher intrinsic 
reactivity can be matched by improving other interactions. Furthermore, it was observed that inhibitors $\mathbf{1 6}$ and 37, both possessing less bulky linkers, exhibited greater reversible inhibition against the BoNT/A LC over compounds that possessed bulkier amide-based linkers (Fig. 2B). We posit that the restricted conformations of the amide bond result in unfavorable trajectory of the reactive centers with respect to Cys165. More flexible alkyl linkers may allow for improved binding interactions and optimal placement of the warhead within the small cleft that presides between Cys165 and the catalytic $\mathrm{Zn}^{2+}$ center. This observation reflects a core advantage of the bifunctional strategy; less reactive warheads can be utilized for covalent modification if they can be effectively directed.

\section{Confirmation of covalent modification of Cys165}

Due to substrate depletion, the linear range of the continuous SNAPtide assay is limited to approximately $50 \mathrm{~min}$. To investigate irreversible inhibition of the BoNT/A LC over a longer incubation time, we employed a conventional method of monitoring covalent kinetics, whereby enzyme and inhibitor are preincubated and aliquots are quenched at given time points and enzyme activity monitored. Thus, compounds 30, 32 and 36 were evaluated for covalent inhibition over the course of $5 \mathrm{~h}$ (Fig. 3A).

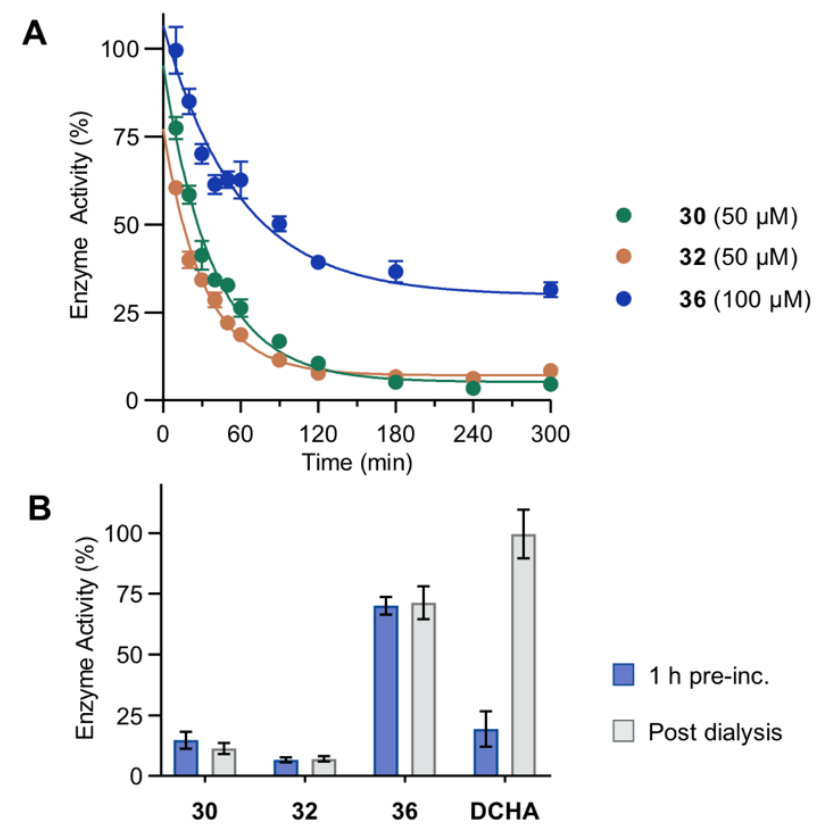

Fig. 3. (A) Preincubation assay showing covalent inhibition over time. (B) Dialysis studies of covalent inhibitors. Inhibitors screened using same conditions as for preincubation assay. Error bars represent $\pm S D, n=3$. See Fig. S4 for full data set.

When incubated at a concentration of $50 \mu \mathrm{M}$ with $500 \mathrm{nM}$ BoNT/A LC, both $\mathbf{3 0}$ and 32 exhibited time-dependent inhibition, reaching a maximum of $\sim 95 \%$ inhibition after $\sim 3 \mathrm{~h}$. Epoxide 36 exhibited considerably slower time-dependent inhibition, reflecting its lower reactivity, reaching a maximum of $\sim 70 \%$ inhibition with $100 \mu \mathrm{M}$ compound after $\sim 8 \mathrm{~h}$ (Fig. S4D). To confirm irreversible inhibition, preincubated enzyme/compound mixtures were exhaustively dialyzed (Fig. 3B), removing excess unreacted inhibitor from the enzyme, and the enzyme activity re-evaluated. BoNT/A LC activity remained unchanged for 30, 32 and 36, indicating an irreversible binding mechanism. DCHA, which was included as a non-covalent control, showed recovery of BoNT/A LC activity after dialysis, confirming its reversible binding mechanism. In 
addition to kinetic analysis of covalent modification, HRMS was used to detect formation of covalent adducts of all bifunctional inhibitors on the BoNT/A LC (See ESI-1, Section 6.2 for raw deconvoluted traces). Compounds exhibiting time-dependent behavior within the continuous assay showed adduct masses consistent with the addition of one labelling event within one hour of incubation with the BoNT/A LC (Fig. S5). The slower-acting epoxide 36 required longer incubation to exhibit significant time-dependent labelling, which was consistent with our kinetic evaluation.

\section{Metal chelating anchor directs the warhead toward Cys165}

To validate the hypothesized mechanism of inhibition, five control compounds were synthesized (Fig. 4A). Compounds 39-41 lack the key hydroxamate anchor and would establish the importance of the MBG in directing the covalent warhead toward Cys165. Compounds $\mathbf{4 2}$ and $\mathbf{4 3}$ were designed as non-covalent versions of compounds $\mathbf{3 0}$ and $\mathbf{3 2}$ respectively, whereby the electrophilic potential of the warhead was replaced with inert functionality.

A

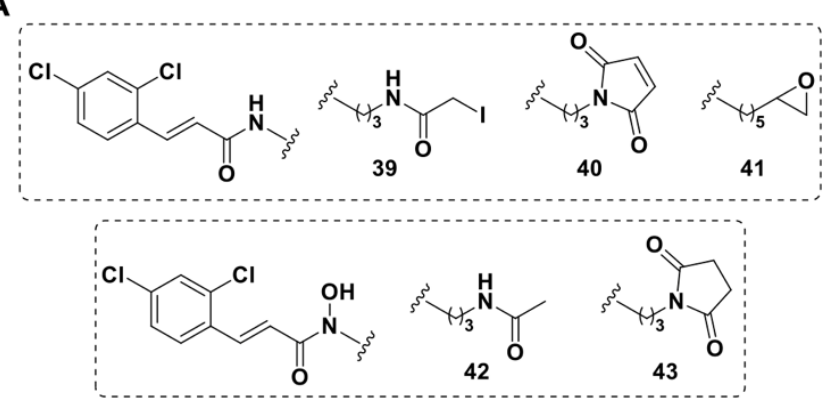

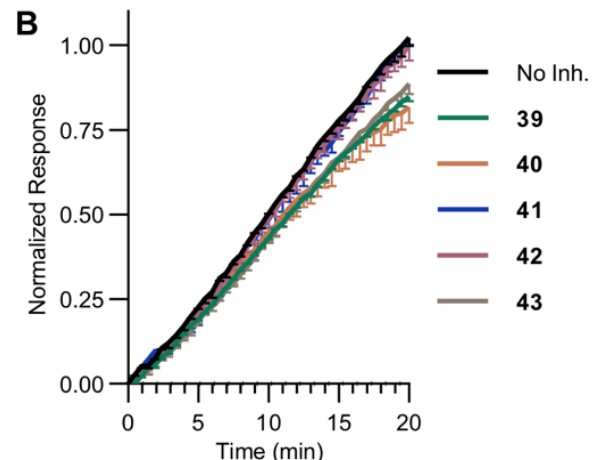

Fig. 4. (A) Control compounds 39-43. (B) Kinetic profiles of control compounds in the continuous assay. Compounds were run at $32 \mu \mathrm{M}$, data was normalized to the maximal fluorescence of the no inhibitor control at $20 \mathrm{~min}$. Error bars represent $\pm \mathrm{SEM}, \mathrm{n}=3$.

Both compounds 39 and $\mathbf{4 0}$ exhibited slight time-dependent inhibition but were drastically reduced when compared to the hydroxamate parent compounds 30 and 32 (Fig. 2C). Moreover, compound 41 showed no time-dependent inhibition. This indicated that the bidentate metal-binding interaction between the hydroxamate and the active-site $\mathrm{Zn}^{2+}$ is fundamental in directing the covalent appendage towards Cys165. As expected, non-covalent controls $\mathbf{4 2}$ and $\mathbf{4 3}$ displayed no activity consistent with covalent inhibition. In addition, both compounds showed poor competitive inhibition, bolstering the earlier hypothesis that sterically bulky linkers and warheads may not allow for optimal binding. To explore the role of Cys165 in covalent bond formation, we expressed a variant of the BoNT/A LC in which Cys165 was replaced with an alanine residue. Compounds 30, 32 and $\mathbf{3 6}$ were evaluated against the C165A variant in the preincubation assay (Fig. S6), all of which exhibited no time-dependent inhibition, outlining the importance of Cys165 in covalent bond formation. Notably, we found that the C165A variant was less active and less stable than the WT, which is in agreement with previous reports. ${ }^{26,28}$ Although it may not be directly involved in the catalytic machinery, Cys 165 likely plays an important structural role for LC activity.

\section{Epoxide 36 possesses superior pharmacological properties}


The promising biochemical data for covalent molecules 30, 32, and $\mathbf{3 6}$ led us to evaluate them against BoNT/A in a cell assay that employs the use of human induced pluripotent stem cell (hiPSC)-derived neurons. ${ }^{36}$ BoNT/A intoxicated neurons were incubated with various concentrations of compound and SNAP-25 cleavage was analyzed by Western blot analysis (Fig. 5A). Compounds 30 and 32 completely abrogated BoNT/A activity at the highest tested concentration $(100 \mu \mathrm{M})$, showing dose dependent behavior. The high $k_{\mathrm{GSH}}$ values of $\mathbf{3 0}$ and 32 (Fig. 2D) suggest that reaction with endogenous GSH may be exhausting the inhibitors, highlighting the need for a less reactive warhead. Furthermore, moderate cell rounding, which is indicative of cytotoxicity, was observed at higher concentrations of $\mathbf{3 0}$ and $\mathbf{3 2}$. Unexpectedly, compound $\mathbf{3 6}$ showed no protection of SNAP-25 cleavage across all tested concentrations, even with longer incubation times. As such, we prepared lysate from primary rat spinal cord (RSC) cells, whereby cell lysis was carried out prior to toxin addition and compound evaluation. In this in vitro model, compound $\mathbf{3 6}$ completely protected SNAP25 cleavage at $100 \mu \mathrm{M}$, though it did not protect at lower tested concentrations. This outlines that the inhibitory behavior of compound $\mathbf{3 6}$ against native BoNT/A LC is somehow attenuated in the cellular environment. We suggest that poor cellular permeability could account for the lack of potency within the hiPSC assay. ${ }^{37}$

One particular class of metalloenzyme, matrix metalloproteinases (MMPs), are involved in governing the structure of the extracellular matrix and are frequently targeted by hydroxamates. ${ }^{38-40}$ To test for crossmetalloenzyme interference, compounds 30, 32 and $\mathbf{3 6}$ were screened against a panel of MMPs to establish their selectivity for the BoNT/A LC (Fig. 5C). In general, compounds 30 and $\mathbf{3 6}$ did not show inhibition against MMPs at the tested concentration. The selectivity imparted by the pendent covalent warhead likely overrides the inherent affinity of the hydroxamate for $\mathrm{Zn}^{2+}$, as there is no corresponding cleft or reactive cysteine residue in the active site of these MMPs. Interestingly, at the tested concentration, compound $\mathbf{3 2}$ inhibited the majority of MMPs by over 50\%. A previous report outlined the use of a photoaffinity probe for the irreversible inhibition of MMP-12, whereby a lysine residue near to the active site was covalently modified. ${ }^{41}$ Maleimides are known to react with various nucleophilic amino acids, ${ }^{42}$ including lysine, and suggests a potential role of $\mathbf{3 2}$ in covalent modification of MMPs. The use of maleimides for the modification of Cys165 in the BoNT/A LC poses potential selectivity issues and further outlines the need for less reactive, more selective warheads.

A
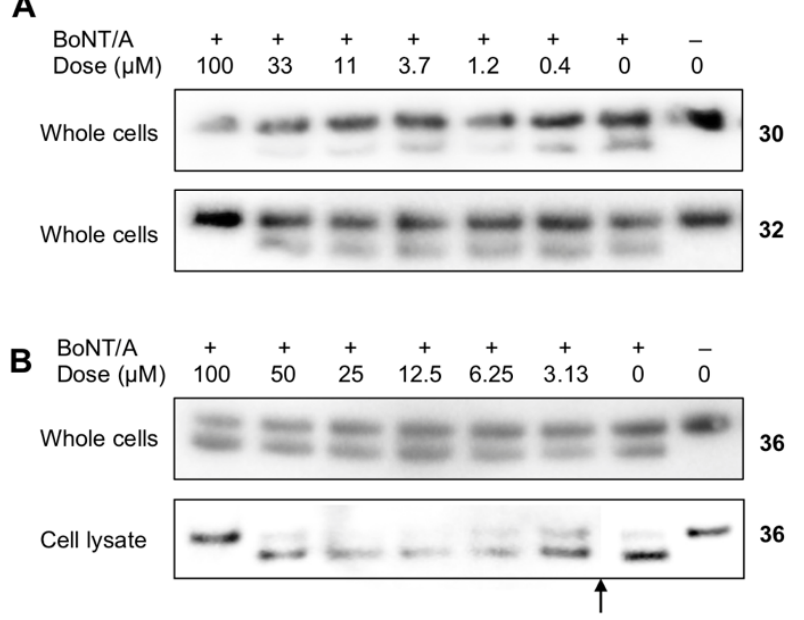
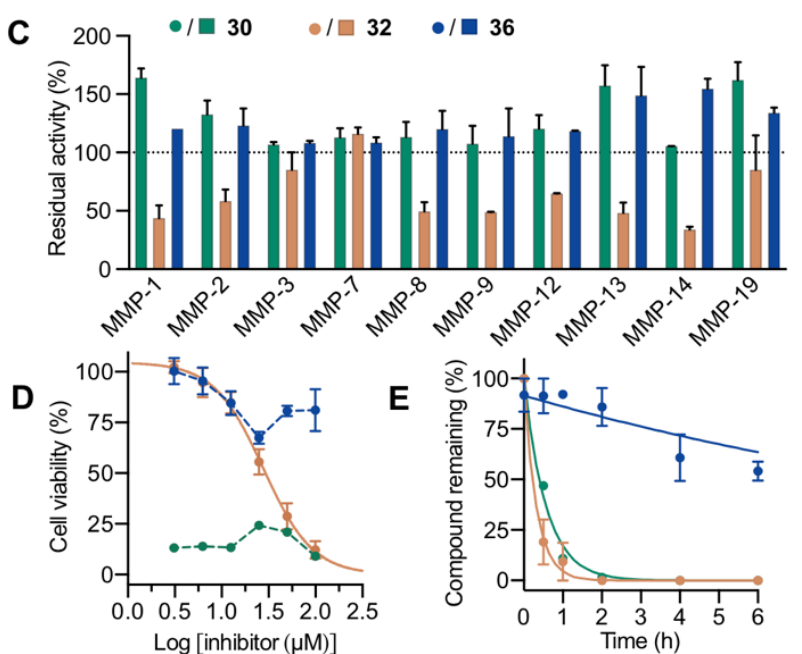

Fig. 5. (A) Western blot evaluation of $\mathbf{3 0}$ and $\mathbf{3 2}$ against post-exposure BoNT/A in hiPCS-derived neurons. BoNT/A cleavage of SNAP25 was measured by quantifying the lower molecular weight band, which corresponds to the SNAP-25 breakdown product, $n=3$. (B) 
Western blot evaluation of $\mathbf{3 6}$ in hiPCS-derived neurons cells and in RSC cell lysate, $n=3$. Arrow depicts image crop of lower compound concentrations. See ESI-1, Appendix 6.1 for full immunoblots. (C) Residual activity of various MMPs in the presence of covalent hits. Compounds tested at $25 \mu \mathrm{M}$. See Table S2 for full data. (D) Cell viability of HEK293 cells post $24 \mathrm{~h}$ compound incubation, $n=3$. See Fig. S7A for viability of additional compounds. (D) Stability assay of compounds in neat human serum at $37^{\circ} \mathrm{C}$, $\mathrm{n}=3$. See Fig. S7B for longer incubation times. Error bars represent \pm SD.

To further explore any off-target potential and toxicity associated with compounds 30, 32 and 36, we evaluated their effect on cell viability in HEK293 cells (Fig. 5D). Control compounds 39, 40, 42 and 43 were also included (Fig. S7A). Compound 30 and iodoacetamide control 39 were toxic across all tested concentrations after $24 \mathrm{~h}$, suggesting off-target alkylation was the likely cause for the observed toxicity in the cellular assay. Both compound $\mathbf{3 2}$ and maleimide control $\mathbf{4 0}$ showed mild toxicity, with $\mathrm{IC}_{50}$ values of $28 \pm 3$ and $6 \pm 2 \mu \mathrm{M}$, respectively. Control compounds $\mathbf{4 2}$ and $\mathbf{4 3}$, which lack the electrophilic warhead, did not exhibit appreciable toxicity indicating that any toxicity imparted by compounds likely stemmed from the warhead and its associated reactivity. This was supported by the observation that compound $\mathbf{3 6}$ was the least toxic, retaining $\sim 75 \%$ cell viability at $100 \mu \mathrm{M}$.

As a further consideration, we evaluated the stability of compounds $\mathbf{3 0}, \mathbf{3 2}$ and $\mathbf{3 6}$ in human serum (Fig. 5E). Compounds 30 and 32 showed rapid degradation, with $t_{1 / 2}<30 \mathrm{~min}$. Compound 36 exhibited excellent serum stability, with $t_{1 / 2}=11 \mathrm{~h}$ (Fig. S7B). These results reflected the trend observed for GSH reactivity and toxicity; more inherently reactive warheads (i.e. MTS, iodoacetamide and maleimide) possess higher potential for off-target effects than the less reactive epoxide warhead of $\mathbf{3 6}$.

\section{Significance}

Classical approaches to covalent inhibition of cysteine proteases utilize the innate high nucleophilicity of the cysteine residue, which often has a low pKa value (4-7) and predominates as the thiolate anion. ${ }^{43-45}$ Electrophilic compounds, known as suicide inactivators, directly modify the active site cysteine and irreversibly interrupt the endogenous mechanism of the enzyme. Contrary to cysteine proteases, metalloproteases employ the use of a metal center to catalyze the cleavage of peptide bonds by association and activation of an active-site water co-factor - no nucleophilic catalytic residues are present in the active site. Direct irreversible interruption of the catalytic machinery involved in metalloproteases has proven difficult, with a limited number of examples reported. ${ }^{46,47}$ Strategies utilizing allosteric modification sites to irreversibly inhibit metalloproteases, namely targeted covalent inhibition, have seen success, ${ }^{41,48,49}$ though the number of such reports remains low relative to the field of small-molecule metalloprotease inhibition. The potential of targeted covalent inhibition is illustrated by its prolific use in kinase inhibitor design, which has boasted several successful drug discovery efforts resulting in FDA-approved covalent kinase inhibitors. ${ }^{50,51}$ These inhibitors have undergone extensive optimization of enzyme/inhibitor interactions such that the electrophilic moieties (i.e. acrylamides) needed for successful covalent reaction possess very little intrinsic reactivity, resulting in exquisite selectivity for their targets. Our work has demonstrated that this approach can be used to leverage the design of selective irreversible inhibitors of the BoNT/A LC. By allowing the use of intrinsically less reactive electrophiles, our bifunctional covalent inhibitor approach has expanded the toolbox of Cys-reactive 
warheads for the BoNT/A LC, allowing the consideration of warheads that possess less off-target inhibition and improved pharmacological potential.

The obtained $k_{\text {inact }} / K_{\mathrm{i}}$ values contrasted with the large difference in GSH reactivity between iodoacetamide- and epoxide-containing compounds illustrate the complex interplay between intrinsic reactivity, binding affinity, and covalent reaction that contributes to the success of these covalent inhibitors. In compound 36, the hydroxamate- $\mathrm{Zn}^{2+}$ interaction acts as a fundamental anchor that is crucial in improving both the effective molarity and proximity of the epoxide to Cys165. Thus, this lowers the entropy of activation and increases the rate of reaction resulting in a selective covalent bond. To further exemplify the importance of the metal-chelating anchor, previous work reported by Garland et al. looked at the use of cysteine-reactive warheads for the covalent modification of Cys 165 in the BoNT/A LC. ${ }^{28}$ Simple scaffolds containing a range of warheads that were devoid of a MBG were shown to have minimal irreversible behavior against the BoNT/A LC. Most importantly, epoxyketone compounds, which possess intrinsically higher reactivity than epoxides alone, did not show modification of Cys165. Only highly reactive selenide-based warheads were capable of modifying Cys $165 .{ }^{28}$ Analysis of the apo active site X-ray crystal structure (PDB 4EJ5) ${ }^{26}$ shows that Cys165 is sequestered deep within a narrow cleft, which in combination with the high predicted pKa of Cys165, would explain the need for highly electrophilic groups. This outlines that the requirements for successful modification of Cys165 using less reactive warheads is contingent upon BoNT/A LC-specific binding functionality, i.e. a metal-binding group, in directing the warhead toward Cys 165.

We envision that this strategy can be utilized amongst other metalloproteases that possess noncatalytic cysteines proximal to the active site metal center. For example, one important class of $\mathrm{Zn}^{2+}$-dependent metalloproteases are the histone deacetylases (HDACs), which are primarily implicated in the epigenetic regulation of the chromatin landscape. ${ }^{52}$ Their prominent role in regulation of gene expression means they are highly sought-after targets for a multitude of diseases, including cancer, inflammatory disease, and metabolic disorders. ${ }^{53}$ The conserved proximal cysteine $\sim 5-7 \AA$ away from the $\mathrm{Zn}^{2+}$ center has been targeted for covalent modification - in 2015, Daniel et al. reported a prodrug of HDAC inhibitor suberoylanilide hydroxamic acid (SAHA) that released SAHA upon modification of the proximal cysteine. Notably, neither species remained covalently tethered to one another, and the thiol-reactive quinine demonstrated rapid reactivity with GSH and non-specific labelling of other cysteines. ${ }^{49} \mathrm{We}$ suggest that the bifunctional strategy could serve to functionalize SAHA and other metal-chelating inhibitor scaffolds, thus allowing the use of less intrinsically reactive warheads to develop irreversible HDAC inhibitors with more favorable pharmacological properties.

\section{Conclusion}

In summary, a comprehensive exploratory search for intrinsically less reactive and more pharmacologically relevant warheads for the covalent modification of Cys165 and subsequent irreversible inhibition of the BoNT/A LC protease was carried out. Covalent inhibition of the BoNT/A LC was challenging given the predicted poor nucleophilicity of Cys165 near the $\mathrm{Zn}^{2+}$-containing active site. Thus, mild electrophiles (i.e. acrylamides and chloroacetamides) furnished no covalent reactivity, making the BoNT/A LC seemingly more difficult to inhibit than other targets reported in literature. ${ }^{54}$ Despite this, covalent labelling 
was achieved using iodoacetamide-, maleimide- and epoxide-containing inhibitors 30-33 and 36. As outlined with the epoxide series, flexible alkyl linkers offered optimal positioning of the warhead to Cys165, resulting in a directed, proximity-driven covalent bond. This was exemplified through the superior covalent inhibition of epoxide $\mathbf{3 6}$ over iodoacetamides $\mathbf{3 0}$ and $\mathbf{3 1}$, despite their huge differences in warhead reactivity. Covalent mechanism of inhibition was confirmed through exhaustive dialysis studies with confirmation of covalent adducts using HRMS. The importance of the bifunctional approach was investigated through synthesis of key control compounds 39-43 with successful covalent modification contingent upon the crucial metal chelating anchor. Modification of Cys165 was confirmed through HRMS and kinetic evaluation of compounds against the $\mathrm{C} 165 \mathrm{~A}$ variant. A trend in GSH reactivity, serum stability, and toxicity was observed, with compound 36 possessing superior pharmacological properties. The drop in potency between enzymatic and cellular assays may be attributed to poor cell permeability rather than off-target reactivity, as exemplified by the RSC lysate assay. We postulate that this bifunctional strategy could be easily translated to other metalloproteases that possess non-catalytic reactive residues near the active site, allowing medicinal chemists to increase the fine margin between potency and toxicity of irreversible covalent inhibitors. 


\section{Author Contributions}

L.D.T, M.E.O, and K.D.J conceptualized the study; L.D.T, A.L.N., and T.S. performed chemical synthesis. L.D.T, A.L.N., and L.L. performed biochemical characterization and analysis of raw data; A.L.N. carried out computational modelling; L.D.T performed mutagenesis and expression of the variant; S.P. and A.L.N. performed cell-based assays; L.D.T. wrote the original draft of the manuscript with input from A.L.N and L.L, and all authors reviewed and edited the final manuscript; E.A.J and K.D.J. acquired funding and supervised the study.

\section{ORCID}

Lewis D. Turner: 0000-0003-0660-8247

Alexander L. Nielsen: $\underline{0000-0003-1195-0143}$

Lucy Lin: 0000-0001-9546-6919

Margaret E. Olson: 0000-0003-1865-9615

Kim D. Janda: $\underline{0000-0001-6759-4227}$

\section{Conflicts of Interest}

There are no conflicts to declare.

\section{Acknowledgments}

We thank Dr. Jiaxing Wang for provision of chemical building blocks, Bill Webb and Elizabeth Billings for technical protein HRMS support, Dr. Joseph Barbieri for kindly supplying the BoNT/A LC, Dr. Lisa Eubanks for mutagenesis guidance, William H. Tepp for production of BoNT/A holotoxin, and Dr. Jason Chen for useful discussions regarding synthesis. This work was supported by the National Institutes of Health grants R01 AI153298 and R21 AI137709 (K.D.J.) and F32 DA044692 (M.E.O.); the Fulbright Scholar Program (A.L.N.); the Natural Sciences and Engineering Research Council of Canada PGSD3-502274 (L.L.) and the Skaggs Institute for Chemical Biology (L.L. and K.D.J.). This is Scripps Research manuscript \#

\section{Associated Content}

The Electronic Supplementary Information (ESI) is available free of charge:

ESI-1: Supplementary figures and tables, biochemical materials and methods, chemical schemes and experimental, full immunoblots, raw deconvoluted HRMS spectra, and compound HPLC chromatograms.

ESI-2: NMR spectra of reported compounds.

\section{Abbreviations}

BoNT/A, botulinum neurotoxin A; CDC, Center for Disease Control and Prevention; DCHA, 2,4dichlorocinnamic hydroxamic acid; ESI, Electronic Supplementary Information; FDA, Food and Drug Administration; FRET, fluorescence resonance energy transfer; GSH, glutathione, HC, heavy chain; HDAC, histone deacetylase; hiPSC, human induced pluripotent stem cell; HRMS, high-resolution mass spectrometry; 
LC, light chain; MBGs, metal-binding groups; MMPs, matrix metalloproteinases; MTS, methanethiosulfonate; MTSEA, (2-aminoethyl) methanethiosulfonate; NC, not calculable; ND, not determined; NSF, Nethylmaleimide sensitive factor; RSC, rat spinal cord; SAHA, suberoylanilide hydroxamic acid; SAR, structure-activity relationship; SNARE, NSF attachment protein factor; SNAP-25, synaptosomal-associated protein-25; THP, tetrahydropyran. 


\section{Notes and references}

1. M. W. Peck, Adv. Microb. Physiol., 2009, 55, 183-265, 320.

2. R. L. Shapiro, C. Hatheway and D. L. Swerdlow, Ann. Intern. Med., 1998, 129, 221-228.

3. M. D. Collins and A. K. East, J. Appl. Microbiol., 1998, 84, 5-17.

4. K. A. Jackson, B. E. Mahon, J. Copeland and R. P. Fagan, Botulinum J, 2015, 3, 6-17.

5. S. S. Arnon, R. Schechter, T. V. Inglesby, D. A. Henderson, J. G. Bartlett, M. S. Ascher, E. Eitzen, A. D. Fine, J. Hauer, M. Layton, S. Lillibridge, M. T. Osterholm, T. O'Toole, G. Parker, T. M. Perl, P. K. Russell, D. L. Swerdlow, K. Tonat and f. t. W. G. o. C. Biodefense, JAMA, 2001, 285, 10591070.

6. P. G. Foran, N. Mohammed, G. O. Lisk, S. Nagwaney, G. W. Lawrence, E. Johnson, L. Smith, K. R. Aoki and J. O. Dolly, J. Biol. Chem., 2003, 278, 1363-1371.

7. M. Oliveira, G. Mason-Buck, D. Ballard, W. Branicki and A. Amorim, Forensic Sci. Int., 2020, 314, 110366.

8. R. B. Lipton, N. L. Rosen, J. Ailani, R. E. DeGryse, P. J. Gillard and S. F. Varon, Cephalalgia, 2016, 36, 899-908.

9. $\quad$ S. Ozcakir and K. Sivrioglu, Clin. Med. Res., 2007, 5, 132-138.

10. X. Yan, J. Lan, Y. Liu and J. Miao, Med Sci Mon Int Med J Exp Clin Res, 2018, 24, 8160-8171.

11. S. M. Farag, M. O. Mohammed, T. A. El-Sobky, N. A. ElKadery and A. K. ElZohiery, JBJS Reviews, 2020, 8, e0119.

12. A. S. o. P. Surgeons, Plastic Surgery Statistics Report, https://www.plasticsurgery.org/documents/News/Statistics/2019/plastic-surgery-statistics-full-report2019.pdf, (accessed 10/05/2020, 2020).

13. E. Yiannakopoulou, Pharmacology, 2015, 95, 65-69.

14. R. Pellizzari, O. Rossetto, G. Schiavo and C. Montecucco, Philos. Trans. R. Soc. Lond. B Biol. Sci., 1999, 354, 259-268.

15. A. Rummel, T. Karnath, T. Henke, H. Bigalke and T. Binz, J. Biol. Chem., 2004, 279, 30865-30870.

16. S. Sun, S. Suresh, H. Liu, W. H. Tepp, E. A. Johnson, J. M. Edwardson and E. R. Chapman, Cell Host Microbe, 2011, 10, 237-247.

17. V. V. Vaidyanathan, K. Yoshino, M. Jahnz, C. Dörries, S. Bade, S. Nauenburg, H. Niemann and T. Binz, J. Neurochem., 1999, 72, 327-337.

18. C. O. Tacket, W. X. Shandera, J. M. Mann, N. T. Hargrett and P. A. Blake, Am. J. Med., 1984, 76, 794-798.

19. L. Lin, M. E. Olson, L. M. Eubanks and K. D. Janda, Acc. Chem. Res., 2019, 52, 2322-2331.

20. L. Lin, L. D. Turner, P. Šilhár, S. Pellett, E. A. Johnson and K. D. Janda, RSC Med. Chem., 2021, DOI: https://doi.org/10.1039/D0MD00320D

21. G. E. Boldt, J. P. Kennedy and K. D. Janda, Org. Lett., 2006, 8, 1729-1732.

22. L. M. Eubanks, M. S. Hixon, W. Jin, S. Hong, C. M. Clancy, W. H. Tepp, M. R. Baldwin, C. J. Malizio, M. C. Goodnough, J. T. Barbieri, E. A. Johnson, D. L. Boger, T. J. Dickerson and K. D. Janda, Proc. Natl. Acad. Sci. U.S.A., 2007, 104, 2602.

23. J. A. Day and S. M. Cohen, J. Med. Chem., 2013, 56, 7997-8007.

24. A. Y. Chen, R. N. Adamek, B. L. Dick, C. V. Credille, C. N. Morrison and S. M. Cohen, Chem. Rev., 2019, 119, 1323-1455.

25. B. Li, S. C. Cardinale, M. M. Butler, R. Pai, J. E. Nuss, N. P. Peet, S. Bavari and T. L. Bowlin, Biorg. Med. Chem., 2011, 19, 7338-7348.

26. E. A. Stura, L. Le Roux, K. Guitot, S. Garcia, S. Bregant, F. Beau, L. Vera, G. Collet, D. Ptchelkine, H. Bakirci and V. Dive, J. Biol. Chem, 2012, 287, 33607-33614.

27. L. Lin, M. E. Olson, T. Sugane, L. D. Turner, M. A. Tararina, A. L. Nielsen, E. K. Kurbanov, S. Pellett, E. A. Johnson, S. M. Cohen, K. N. Allen and K. D. Janda, J. Med. Chem., 2020, 63, 1110011120.

28. M. Garland, B. M. Babin, S.-I. Miyashita, S. Loscher, Y. Shen, M. Dong and M. Bogyo, ACS Chem. Biol., 2019, 14, 76-87.

29. M. Lo Conte, J. Lin, M. A. Wilson and K. S. Carroll, ACS Chem. Biol., 2015, 10, 1825-1830.

30. N. R. Silvaggi, G. E. Boldt, M. S. Hixon, J. P. Kennedy, S. Tzipori, K. D. Janda and K. N. Allen, Chem. Biol., 2007, 14, 533-542.

31. M. A. Walker, Tetrahedron Lett., 1994, 35, 665-668. 
32. C. R. Søndergaard, M. H. M. Olsson, M. Rostkowski and J. H. Jensen, J. Chem. Theory Comput., 2011, 7, 2284-2295.

33. Y. Kim, S. O. Ho, N. R. Gassman, Y. Korlann, E. V. Landorf, F. R. Collart and S. Weiss, Bioconjug. Chem., 2008, 19, 786-791.

34. C. Smythe, J. Biol. Chem, 1936, 114, 601-612.

35. A. Böhme, D. Thaens, A. Paschke and G. Schüürmann, Chem. Res. Toxicol., 2009, 22, 742-750.

36. S. Pellett, W. H. Tepp, C. M. Clancy, G. E. Borodic and E. A. Johnson, FEBS Lett., 2007, 581, 4803-4808.

37. P. Silhár, L. M. Eubanks, H. Seki, S. Pellett, S. Javor, W. H. Tepp, E. A. Johnson and K. D. Janda, J. Med. Chem., 2013, 56, 7870-7879.

38. G. Murphy and H. Nagase, Mol. Aspects Med., 2008, 29, 290-308.

39. J. A. Jacobsen, J. L. Major Jourden, M. T. Miller and S. M. Cohen, Biochim. Biophys. Acta, 2010, 1803, 72-94.

40. J. M. Cathcart and J. Cao, Front. Biosci. (Landmark Ed), 2015, 20, 1164-1178.

41. A. S. Dabert-Gay, B. Czarny, E. Lajeunesse, R. Thai, H. Nagase and V. Dive, Bioconjug. Chem., 2009, 20, 367-375.

42. N. E. Sharpless and M. Flavin, Biochemistry, 1966, 5, 2963-2971.

43. F. Hofer, J. Kraml, U. Kahler, A. S. Kamenik and K. R. Liedl, J. Chem. Inf. Model., 2020, 60, 30303042 .

44. T. K. Harris and G. J. Turner, IUBMB Life, 2002, 53, 85-98.

45. J. C. Powers, J. L. Asgian, Ö. D. Ekici and K. E. James, Chem. Rev., 2002, 102, 4639-4750.

46. M. M. Bernardo, S. Brown, Z. H. Li, R. Fridman and S. Mobashery, J. Biol. Chem., 2002, 277, 11201-11207.

47. S. Brown, M. M. Bernardo, Z.-H. Li, L. P. Kotra, Y. Tanaka, R. Fridman and S. Mobashery, J. Am. Chem. Soc., 2000, 122, 6799-6800.

48. N. Sin, L. Meng, M. Q. W. Wang, J. J. Wen, W. G. Bornmann and C. M. Crews, Proc. Natl. Acad. Sci. U.S.A., 1997, 94, 6099-6103.

49. K. B. Daniel, E. D. Sullivan, Y. Chen, J. C. Chan, P. A. Jennings, C. A. Fierke and S. M. Cohen, J. Med. Chem., 2015, 58, 4812-4821.

50. D. Kazandjian, G. M. Blumenthal, W. Yuan, K. He, P. Keegan and R. Pazdur, Clin. Cancer. Res., 2016, 22, 1307-1312.

51. H. Singh, A. J. Walker, L. Amiri-Kordestani, J. Cheng, S. Tang, P. Balcazar, K. Barnett-Ringgold, T. R. Palmby, X. Cao, N. Zheng, Q. Liu, J. Yu, W. F. Pierce, S. R. Daniels, R. Sridhara, A. Ibrahim, P. G. Kluetz, G. M. Blumenthal, J. A. Beaver and R. Pazdur, Clin. Cancer. Res., 2018, 24, 34863491.

52. G. Milazzo, D. Mercatelli, G. Di Muzio, L. Triboli, P. De Rosa, G. Perini and F. M. Giorgi, Genes, 2020, 11, 556.

53. D. F. Tough, P. P. Tak, A. Tarakhovsky and R. K. Prinjha, Nat. Rev. Drug Discov., 2016, 15, 835853.

54. E. Resnick, A. Bradley, J. Gan, A. Douangamath, T. Krojer, R. Sethi, P. P. Geurink, A. Aimon, G. Amitai, D. Bellini, J. Bennett, M. Fairhead, O. Fedorov, R. Gabizon, J. Gan, J. Guo, A. Plotnikov, N. Reznik, G. F. Ruda, L. Díaz-Sáez, V. M. Straub, T. Szommer, S. Velupillai, D. Zaidman, Y. Zhang, A. R. Coker, C. G. Dowson, H. M. Barr, C. Wang, K. V. M. Huber, P. E. Brennan, H. Ovaa, F. von Delft and N. London, J. Am. Chem. Soc., 2019, 141, 8951-8968. 


\section{Graphical abstract}

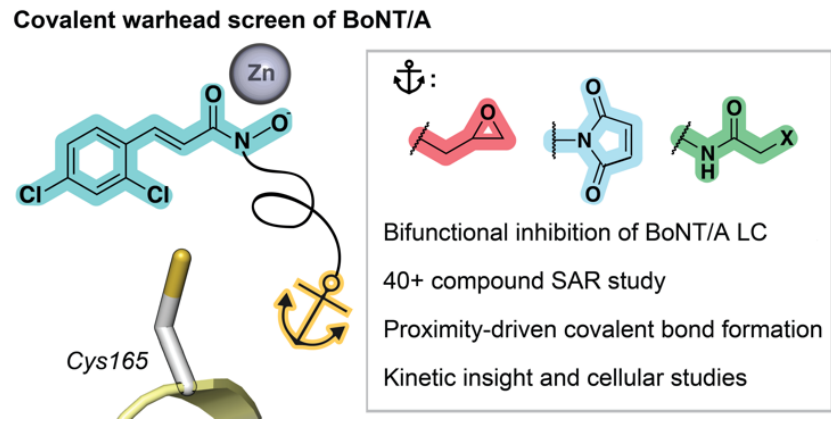

